\title{
EVALUACIÓN DE DISTINTOS NIVELES DE COMPRENSIÓN LECTORA EN ESTUDIANTES MEXICANOS DE PSICOLOGÍA
}

\author{
Yolanda Guevara Benítez ${ }^{*}$, Jorge Guerra García ${ }^{1}$, Ulises Delgado Sánchez¹, Claudia Flores Rubí1 \\ ${ }^{1}$ Universidad Nacional Autónoma de México-FES Iztacala
}

Recibido, noviembre 6/2013

Concepto de evaluación, abril 3/2014

Aceptado, mayo 9/2014
Referencia: Guevara Benítez, Y., Guerra García, J., Delgado Sánchez, U. \& Flores Rubí, C. (2014). Evaluación de distintos niveles de comprensión lectora en estudiantes mexicanos de psicología. Acta Colombiana de Psicología, 17 (2), pp. 113-121. DOI:10.14718/ACP.2014.17.2.12

Resumen

Actualmente no se cuenta con un sistema de evaluación de las competencias lectoras de los alumnos universitarios mexicanos. Esta investigación evaluó habilidades en cinco niveles de comprensión lectora, con un instrumento validado. Participaron 570 estudiantes de la Carrera de Psicología, de los diferentes semestres, con edad promedio de 19.9 años. Se aplicó el instrumento grupalmente. La prueba arrojó un porcentaje promedio general de $66 \%$ de respuestas correctas. Los tres niveles de comprensión que mostraron porcentajes inferiores a 50\% fueron: literal, inferencial y crítico; mientras que los niveles de organización de la información y apreciativo exhibieron porcentajes por encima del 70\%. El ANOVA indicó diferencias $(p<.05)$ en cuatro niveles de comprensión, a favor de los alumnos de los primeros semestres. Se discuten los hallazgos a la luz de investigaciones previas y de sus implicaciones formativas.

Palabras clave: comprensión lectora, niveles de comprensión, texto académico, evaluación, estudiantes universitarios.

\section{ASSESSMENT OF DIFFERENT LEVELS OF READING COMPREHENSION IN MEXICAN PSYCHOLOGY STUDENTS}

\begin{abstract}
No system for evaluating reading competences of Mexican university students is currently available. This research assessed five levels of skills in reading comprehension, using a validated instrument. Participants were 570 Psychology students, attending different semesters, with a mean age of 19.9 years. The instrument was administered in groups. The test yielded an overall average of $66 \%$ correct responses. The three levels of comprehension that showed percentages lower than $50 \%$ were: literal, inferential and critical, while the information organization level and the appreciative level exhibited percentages above $70 \%$. The ANOVA indicated differences $(\mathrm{p}<.05)$ in four levels of reading comprehension in favor of students in the first semester. Findings are discussed in light of previous research and their educational implication.

Key words: reading comprehension, comprehension levels, academic text, evaluation, college students.

\section{AVALIAÇÃO DE DIFERENTES NÍVEIS DE COMPREENSÃO LEITORA EM ESTUDANTES MEXICANOS DE PSICOLOGIA}

Resumo

Atualmente não se conta com um sistema de avaliação de as competências leitoras dos estudantes universitários mexicanos. Esta pesquisa avaliou habilidades em cinco niveles de compreensão leitora, com um instrumento validado. Participaram 570 estudantes de a Carrera de Psicologia, de os diferentes semestres, com idade média de 19.9 anos. Aplicou-se o instrumento grupalmente. O teste mostrou uma porcentagem média general de $66 \%$ de respostas corretas. Os três níveis de compreensão que mostraram porcentagens inferiores a 50\% foram: literal, inferencial e crítico; enquanto que os níveis de organização da informação e apreciativo exibiram porcentagens acima de 70\%. O ANOVA indicou diferenças $(p<.05)$ em quatro níveis de compreensão, a favor dos estudantes dos semestres. Discutem-se descobrimentos à luz de pesquisas anteriores e de suas implicações formativas. Palavras chave: comprensión lectora, niveles de comprensión, texto académico, avaliação, estudiantes universitários.
\end{abstract}

* Av. De los Barrios no. 1, Col. Los Reyes Iztacala. Tlalnepantla, Estado de México. Código Postal 54090. Teléfono: (55) 53931333 ext.39762. yolaguevara@hotmail.com 


\section{INTRODUCCIÓN}

Los estudiosos de la comprensión lectora están de acuerdo en considerarla como una competencia indispensable para el aprendizaje de conocimientos, habilidades, actitudes y competencias específicas (Díaz, 2006; Walter, 2004). Por ello, ha adquirido relevancia en todo el mundo, llegando a ser una de las competencias genéricas a las que se les confiere más importancia a nivel formativo. Ejemplo de esto es su inclusión en un apartado específico dentro de las evaluaciones internacionales y nacionales sobre el desempeño de las instituciones educativas y de sus estudiantes en diversos niveles formativos.

Además, dentro de los planes curriculares-que se basan en el desarrollo de competencias-se consideran como objetivos académicos diversos componentes de la comprensión lectora. Entre tales componentes están la realización de síntesis, inferencias y análisis crítico de contenidos de discursos y lecturas, así como la identificación y jerarquización de ideas (Goscinski et al., 2005; Quintana, Raccoursier, Sánchez, Sidler, \& Toirkens, 2007; Secretaría de Educación Pública, 2011, 2013; Sumsion \& Goodfellow, 2004). Lo anterior se basa en la consideración de que la adquisición y uso generalizado de la comprensión lectora es indispensable en todos los niveles educativos - especialmente en los superiores-porque una gran parte de la formación de los alumnos se lleva a cabo a través de la lectura de textos. De hecho, autores como Irigoyen, Acuña y Jiménez (2010) señalan la necesidad de que un objetivo prioritario en las universidades debería ser enseñar a sus alumnos competencias con las cuales puedan aprender por sí mismos, incluyendo la lectura comprensiva.

A pesar de lo anterior, en el contexto del Sistema Educativo Mexicano se han reportado serios problemas formativos relacionados con esta competencia. Sánchez y Andrade (2009) retoman los resultados reportados por el Instituto Nacional para la Evaluación de la Educación (INEE). Enfatizan el hecho de que tales reportes indican que sólo el 32\% de los alumnos de sexto grado de educación primaria obtuvo un nivel satisfactorio de lectura. Agregan que los Exámenes de Calidad y Logro Educativo (Excale) aplicados por el INEE en 2008 a los estudiantes nacionales de tercer grado de secundaria, reportaron que el $72 \%$ de los estudiantes no pudo interpretar información proveniente de diferentes partes del texto en ensayos y textos científicos, así como el contenido y organización de las ideas, argumentos, contraargumentos y elementos persuasivos. Estos alumnos tampoco pudieron reconocer las oraciones temáticas de artículos de opinión y de divulgación, la trama de cuentos clásicos, ni relacionar la información del texto con la que se ofrece en imágenes, tablas y gráficas.
Por su parte, las evaluaciones llevadas a cabo por la Organización para la Cooperación y el Desarrollo Económico (OCDE, 2007), a través de las pruebas PISA (Programme for International Student Assessement), reportan que menos del 7\% de los alumnos mexicanos evaluados pueden catalogarse como buenos lectores, y que tales resultados no mejoraron de la evaluación aplicada en el año 2003 a la aplicada en 2006. Dado que estas pruebas evalúan estudiantes de 15 años de edad, en 42 países, es posible saber que México ocupa uno de los últimos lugares en el desempeño estudiantil de diversas áreas académicas, en especial, en la comprensión lectora. Tales resultados constituyen un panorama desalentador respecto a los logros de la educación básica mexicana. Mujica, Guido y Mercado (2011) señalan que las bajas puntuaciones de los alumnos mexicanos en tales evaluaciones guardan una estrecha relación con los bajos índices de actividades lectoras en la población, reportados por la Encuesta Nacional de Lectura, los cuales indican que $33.3 \%$ de los mexicanos nunca ha leído un libro y que el promedio de libros leídos anualmente, per cápita, es de 2.6 en el país.

Dado que el problema de la baja comprensión lectora persiste en diversos niveles de formación básica, es lógico suponer que los alumnos que ingresan a la universidad puedan carecer de algunas habilidades lectoras, y por tanto, verse limitados en la consecución de los objetivos curriculares de la licenciatura. Ello puede implicar, incluso, que los estudiantes que egresan de la universidad pueden tener obstáculos para insertarse exitosamente en el mercado laboral competitivo. Desafortunadamente, no se cuenta con un sistema de evaluación de las competencias lectoras de los alumnos universitarios, que pueda dar información precisa sobre cuáles aspectos han sido desarrollados adecuadamente y cuáles son deficitarios en los estudiantes que ingresan, en los que cursan los diversos semestres y en los que egresan de las universidades.

Algunos autores han aplicado evaluaciones informales de la comprensión lectora en estudiantes universitarios mexicanos (Rizo, 2004; Sánchez \& Acle, 2001; Zarzosa, 1997). Sus reportes coinciden en señalar que uno de los problemas de estos alumnos es su dificultad para seleccionar la información importante de los textos, lo cual está relacionado con su capacidad de utilizar estrategias efectivas para extraer las ideas principales. Similarmente, Echevarría y Gastón (2000) reportan problemas de comprensión en estudiante universitarios, en relación con un texto expositivo-argumentativo, señalando que las dificultades se presentaron en la selección y jerarquización de las ideas principales; sólo el $4 \%$ de los alumnos participantes en dicho estudio captó seis o más ideas principales, de las 
ocho que contenía el texto, mientras que el $46 \%$ de ellos sólo captó dos.

En concordancia, Calderón y Quijano (2010) reportan un bajo nivel de comprensión lectora en estudiantes de los primeros años de las carreras de Derecho y Psicología de una universidad colombiana, mientras que Cisneros, Olave y Rojas (2010) reportan que las principales dificultades involucradas en la comprensión lectora de los alumnos colombianos están relacionadas directamente con el uso de la inferencia, e incluyen dificultades para definir los propósitos del autor y del texto, para identificar ideas principales, y para realizar la lectura relacionando significados, entre otras.

Vera, Estévez y Ayón (2010) realizaron una investigación con alumnos de tres instituciones de educación superior en el noroeste de México. Tomaron como base los resultados de la aplicación del instrumento del Proyecto Tuning en América Latina, relativo a las competencias para el trabajo, y les preguntaron sobre el nivel de logro de dichas competencias, relacionándolas con lo que cursan en la carrera universitaria. Entre los resultados más importantes sobresale que los estudiantes de las tres universidades reportaron un desarrollo insuficiente de competencias relacionadas con los procesos de aprendizaje, como son crítica, autocrítica, abstracción, análisis y síntesis. Los alumnos atribuyeron sus bajos desempeños a la escasa importancia que ellos mismos les confieren a esas habilidades, así como al hecho de que los programas académicos de sus universidades no están dirigidos a fomentarlas.

Tales informes marcan ciertas pautas sobre los aspectos que es necesario evaluar respecto a los niveles de comprensión lectora desarrollados por estudiantes universitarios en distintos momentos de su formación profesional. Sin embargo, es necesario contar con estudios sistemáticos que utilicen instrumentos válidos y confiables para medir distintos aspectos (niveles) de comprensión lectora y poder realizar estudios comparativos y de proceso. Contar con tales instrumentos permitirá dirigir estrategias efectivas para el desarrollo de diferentes competencias lectoras que habiliten a los alumnos para un pleno aprovechamiento de sus cursos universitarios. Una herramienta para tal fin es proporcionada por Pérez (2005), al definir la comprensión lectora como el proceso mediante el cual el lector, a través del uso de diversas estrategias, interactúa con el texto. Este autor puntualiza cinco niveles de comprensión lectora.

El nivel de comprensión literal se aprecia cuando el lector puede reconocer y recordar, directamente del texto, las ideas tal y como las expresa el autor. El de reorganización de la información se distingue cuando el lector ordena las ideas mediante procesos de clasificación y síntesis, cuando reseña o sintetiza la lectura de un texto con sus propias palabras, o cuando lo expresa gráficamente a través del uso de conceptos vinculados por símbolos que indican relaciones, jerarquías, etcétera. El nivel inferencial se distingue cuando el lector agrega elementos que no están en el texto, para relacionarlo con sus experiencias personales o para deducir ideas que no están explícitas en el escrito, posibilitando de esta manera su interpretación. El nivel crítico se observa cuando el lector utiliza procesos de valoración acerca del contenido del texto, para lo cual necesita establecer una relación entre lo que dice el escrito y el conocimiento previo que tiene sobre el tema, para luego evaluar las afirmaciones del autor contrastándolas con las propias; este nivel supone comprender diversos modos de interpretar un texto. Por último, el nivel apreciativo se observa cuando el lector puede expresar comentarios emotivos, estéticos o de contenido sobre el texto consultado; o cuando emite juicios sobre el estilo literario o sobre las características del lenguaje que utiliza el autor, por ejemplo, el empleo de la ironía, del humor, del doble sentido, etcétera.

El reconocimiento de dichos niveles de comprensión lectora implica que las evaluaciones de esta competencia han de incluir la medición de todos esos niveles, y no circunscribirse a alguno de ellos. Al respecto, diversos autores (Arroyo, Morales, Silva, Canales, \& Carpio, 2011; Backhoff, Sánchez, Peón, \& Andrade, 2010; Irigoyen, Acuña, \& Jiménez, 2011; Llorens et al., 2011) han expresado una serie de críticas a las pruebas de comprensión lectora que suelen aplicarse a los estudiantes en distintos escenarios escolares. Sus principales argumentos se relacionan con el hecho de que, en su mayoría, son pruebas de opción múltiple, por lo que la evaluación de la comprensión lectora se circunscribe a un tipo o nivel de la misma. Una prueba de opción múltiple evalúa, regularmente, un nivel de comprensión literal. De igual forma, Bazán, Castañeda, Macotela y López (2004) señalan que la elaboración y uso de instrumentos para la evaluación de desempeños deben dirigirse de tal manera que los instrumentos recojan evidencias confiables acerca de las propiedades y atributos definidos teóricamente, y que es necesario evaluar el grado de dominio en actividades de lectura, incluyendo suposiciones, inferencias y deducciones.

Entre los instrumentos válidos y confiables que pueden ser aplicados para evaluar la comprensión lectora, se encuentra el desarrollado por Sánchez, Grajales y García (2011), que utiliza siete textos y una serie de pruebas escritas. A pesar de su sistematicidad, la aplicación de este instrumento puede representar mucho tiempo de aplicación. Por ello, Guerra y Guevara (2013) llevaron a cabo un proceso de conformación y validación del Instrumento para medir Comprensión Lectora en Alumnos Universitarios (ICLAU), que toma en consideración los cinco niveles definidos por Pérez (2005). El instrumento incorpora, además de un texto y unos reactivos relacionados con cada nivel de comprensión lectora, 
herramientas novedosas, derivadas de avances metodológicos recientes en el proceso de evaluación de la comprensión, en particular el uso de rúbricas para calificar las características de las respuestas que los alumnos dan ante preguntas abiertas (Ramos, 2006).

El objetivo de la presente investigación fue evaluar los niveles de comprensión lectora en una muestra de estudiantes universitarios de distintos semestres escolares de la Carrera de Psicología, a partir del instrumento ICLAU, así como observar si existían diferencias en los porcentajes promedio de respuestas correctas de los participantes, considerando cada nivel de comprensión, y en función del semestre académico que cursan los alumnos.

Se planteó la siguiente hipótesis: Habrá diferencias en los puntajes de respuestas correctas de los alumnos, dependiendo del nivel de comprensión lectora evaluado, así como del semestre que estén cursando.

\section{MÉTODO}

El diseño del estudio fue de tipo no experimental, transversal, descriptivo y comparativo.

\section{Participantes}

La población universo estuvo definida por la totalidad de los grupos inscritos durante el ciclo escolar 2013-1, en la Carrera de Psicología de una universidad pública ubicada en el Estado de México. Los semestres en curso correspondieron al primero, tercero, quinto y séptimo. Se consideró como unidad de análisis el grupo escolar en el que estaban inscritos los participantes, del turno matutino y vespertino. Se utilizó un muestreo probabilístico estratificado de tipo proporcional, considerando semestre y turno (Méndez, Namihira, Moreno, \& Sosa, 2006). El total de participantes fue de 570 alumnos, distribuidos en 19 grupos de ambos turnos, inscritos en los cuatro semestres escolares. La edad promedio de los alumnos fue de 19.9 años $(\mathrm{DE}=2.50)$. La distribución por sexo fue de $28 \%$ para el masculino y $72 \%$ para el femenino. En relación con su estado civil, 93\% eran solteros y $3.5 \%$ casados.

\section{Variable}

Se retomó la definición de comprensión lectora de Pérez (2005), quien la considera como el proceso mediante el cual el lector, a través del uso de diversas estrategias, interactúa con el texto en distintos niveles de competencia.

\section{Instrumento}

Se utilizó el Instrumento para medir Comprensión Lectora en Alumnos Universitarios (ICLAU), que consta de un texto de tipo expositivo-argumentativo denominado "La evolución y su historia", extraído de Cela y Ayala (2001), el cual es una narración de 965 palabras cuyo contenido explica la evolución biológica, haciendo énfasis en sus causas y procesos, con un estilo de redacción que permite una lectura ágil y amena. Además, el instrumento contiene una serie de reactivos para evaluar los cinco niveles de comprensión descritos arriba. Su proceso de conformación y validación con estudiantes universitarios mexicanos fue reportado por Guerra y Guevara (2013), quienes aclaran que se optó por este escrito debido a que se refiere a un tema general de las ciencias biológicas y, por tanto, no está vinculado de forma directa con la psicología. Se asumió que el conocimiento previo que pudieran tener los alumnos sobre este tema sería similar en todos ellos, debido a los programas académicos de educación básica y media superior en los cuales fueron formados; por tanto, no se podía considerar que éste fuera un factor clave que influyera en los niveles mostrados de comprensión lectora. Los aspectos validados en cada reactivo del instrumento fueron: redacción, pertinencia y adecuación, en todos los elementos incorporados, incluyendo preguntas, opciones de respuesta, rúbricas y criterios de logro de cada nivel de comprensión lectora a evaluar.

\section{Procedimiento}

La aplicación del instrumento a los alumnos participantes se llevó a cabo en un aula bien iluminada y ventilada, de aproximadamente $6 \mathrm{X} 4$ metros, en donde toman sus clases regularmente. Dicha aplicación se realizó en una sesión grupal de aproximadamente una hora de duración, para cada uno de los grupos escolares por separado.

$\mathrm{Al}$ inicio de la sesión se explicó brevemente a cada grupo de alumnos en qué consistía la investigación. Se le dijo que se estaba llevando a cabo un estudio relativo a los estudiantes y los planes de estudio de la Carrera, para lo cual se les solicitaba que contestaran una serie de preguntas contenidas en tres instrumentos, cuyos resultados no repercutirían en las evaluaciones de ninguna de las asignaturas que estaban cursando. Se les aclaró que no estaban obligados a participar y que si decidían incorporarse voluntariamente al estudio firmarían un consentimiento informado antes de proceder a la aplicación de los instrumentos; también se aclaró que todos los datos proporcionados se mantendrían en absoluta confidencialidad.

A los estudiantes que aceptaron participar se les proporcionaron las instrucciones específicas para contestar el instrumento, y recibieron por escrito dicho instrumento y la carta de consentimiento informado. Cada alumno entregaba sus hojas cuando concluía, y salía del aula. 


\section{Calificación y captura de datos}

Se llevó a cabo la calificación de todas las pruebas aplicadas, tomando en consideración los criterios definidos para cada reactivo del instrumento, relacionados con la definición de los niveles de comprensión lectora. Cada prueba fue calificada por dos evaluadores y ambos debían llegar a un acuerdo con respecto a la calificación que sería registrada; en caso de un desacuerdo persistente entre los evaluadores, se podía recurrir a una tercera opinión, lo cual no fue necesario. Las calificaciones obtenidas fueron capturadas en una base de datos del programa SPSS Versión 15.0 para obtener porcentajes promedio de respuestas correctas, para cada nivel. Con las calificaciones se aplicó un análisis ANOVA de un factor para observar las posibles diferencias en los niveles de comprensión lectora del texto académico (ICLAU) entre los estudiantes inscritos en cada semestre, considerando sus puntajes obtenidos en cada nivel de comprensión de la prueba.

\section{RESULTADOS}

El análisis de los resultados se presenta en términos de los porcentajes obtenidos por los estudiantes de los cuatro semestres en el total del instrumento de comprensión lectora, y en cada uno de los cinco niveles de comprensión que se evaluaron. También se presentan los resultados que en el ANOVA mostraron diferencias estadísticamente significativas durante la comparación de los puntajes de los alumnos.

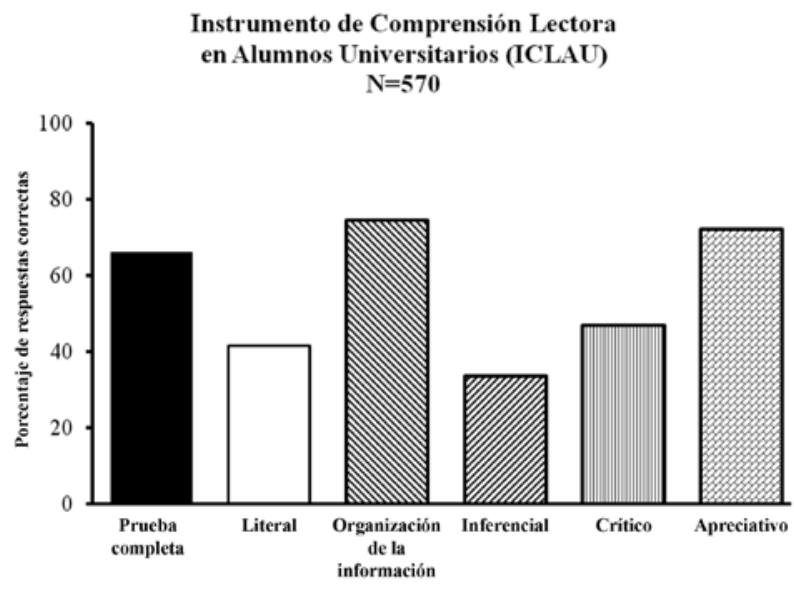

Figura 1. Muestra los porcentajes de respuestas correctas obtenidos por los alumnos de la muestra total en la prueba completa de comprensión lectora del texto académico (ICLAU), y en cada nivel de comprensión evaluado.

La figura 1 presenta los resultados de la aplicación del Instrumento para medir Comprensión Lectora en Alumnos
Universitarios. La prueba global, considerando las preguntas ubicadas para evaluar los cinco niveles de comprensión, arrojó un porcentaje promedio general de $66 \%$ de respuestas correctas. Los tres tipos de comprensión que produjeron porcentajes inferiores a 50 fueron el nivel literal, el inferencial y el crítico; el menor porcentaje se presentó en el nivel inferencial. Por arriba del $70 \%$ se presentaron el nivel de organización de la información y el apreciativo.

La figura 2 muestra los porcentajes promedio de respuestas correctas obtenidos por los alumnos de cada semestre académico, considerando el puntaje total y el de cada nivel de comprensión evaluado. Las diferencias en el porcentaje de respuestas correctas entre los alumnos de los distintos semestres no rebasan el 7\%. En el total del instrumento todos los alumnos mostraron una ejecución alrededor del $66 \%$; en el nivel literal alrededor del $41 \%$, y en los niveles siguientes, $75 \%, 33 \%, 47 \%$ y $72 \%$, respectivamente. Estos datos por semestre se corresponden completamente con los porcentajes de la muestra total, presentados en la figura 1.

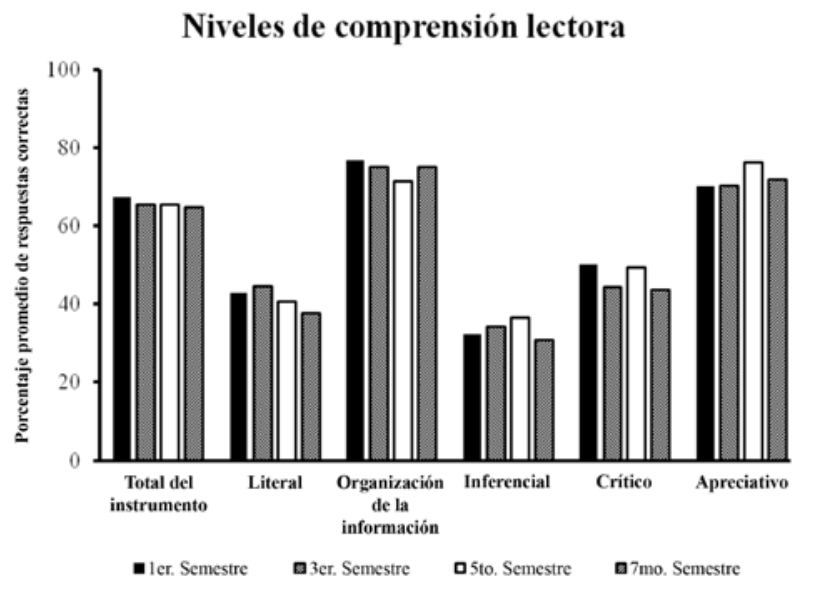

Figura 2. Muestra el porcentaje promedio de respuestas correctas obtenidas por los alumnos de cada semestre académico, en el total del instrumento de comprensión lectora y en cada nivel de comprensión.

El análisis ANOVA de un factor, realizado para observar las posibles diferencias estadísticas en los niveles de comprensión lectora del texto académico (ICLAU) entre los estudiantes inscritos en cada semestre, arrojó diferencias significativas, al nivel de $p<.05$, en cuatro tipos o niveles de comprensión: literal, inferencial, crítico y apreciativo. Para conocer a favor de qué semestre académico fueron tales diferencias estadísticas, se utilizaron las pruebas post hoc de Games-Howell y de Bonferroni, tomando en cuenta los resultados de la prueba de homogeneidad de Levene; si ésta arrojaba diferencias significativas, se procedía a utilizar la 
primera prueba; en caso contrario, se utilizaría la segunda. Se utilizó la prueba de Games-Howell, dado que es poderosa (es pequeña la probabilidad de cometer el error tipo II) y exacta para muestras con tamaños desiguales; y la prueba de Bonferroni, porque minimiza la posibilidad de cometer error tipo I, y porque es poderosa cuando el número de comparaciones que se realizan es pequeño (Field, 2000).

Como se puede observar en la tabla 1, en el nivel de comprensión literal, las diferencias en el promedio de las puntuaciones de los alumnos de primer semestre con respecto a los alumnos de séptimo fueron a favor de los primeros, así como los de tercero fueron significativamente mayores con respecto a los de séptimo semestre. En tanto que, en la comprensión de tipo inferencial y en la apreciativa, los alumnos de quinto semestre tuvieron puntuaciones mayores que los de séptimo y los de primero. Finalmente, en la comprensión crítica, los alumnos de primer semestre obtuvieron puntuaciones mayores que los de tercero. Las restantes comparaciones entre los semestres no mostraron diferencias estadísticamente significativas.

Las diferencias entre las medias obtenidas por los alumnos de los distintos semestres, en cada nivel de comprensión evaluado, se pueden resumir así: en el nivel literal los alumnos de primer y tercer semestre tuvieron un mayor número de respuestas correctas $(M=0.86 ; M=0.89$, respectivamente) que los de séptimo $(M=0.75)$. En el nivel inferencial, los estudiantes del quinto semestre tuvieron un mayor puntaje $(M=0.73)$ que los de séptimo $(M=0.62)$ y en el nivel apreciativo, un mayor puntaje $(M=2.28)$ que los de primer semestre $(M=2.10)$. Por último, en el nivel crítico, los del primer semestre se desempeñaron mejor $(M=3.0)$ que los del tercero $(M=2.67)$.

\section{DISCUSIÓN}

Los resultados del presente estudio son sugerentes, aunque no concluyentes. No puede decirse que son representativos de todos los alumnos mexicanos que estudian psicología, ni de todas las competencias lectoras, pero sí aportan datos para ilustrar aspectos importantes en relación con el tema.

Los resultados obtenidos a través de la aplicación del Instrumento para evaluar Comprensión Lectora en Alumnos Universitarios (ICLAU) permitieron observar que los alumnos de la muestra estudiada tuvieron un bajo desempeño en esta competencia, particularmente en los niveles de comprensión literal, inferencial y crítico, lo cual guarda cierta concordancia con lo arrojado por evaluaciones informales realizadas con estudiantes universitarios (Calderón \& Quijano, 2010; Rizo, 2004; Sánchez \& Acle, 2001; Zarzosa, 1997).

También resultan concordantes con lo señalado por autores como Ugarriza (2006) y Amador y Alarcón (2006), respecto a las recurrentes quejas de los profesores universitarios por el hecho de que sus alumnos presentan habilidades insuficientes en la comprensión, análisis y síntesis de los textos sobre los cuales deben basar el desarrollo de su formación académica profesional. Aspecto que se traduce en bajas expectativas de muchos profesores con respecto a las posibilidades de los alumnos para desarrollarse académicamente, así como en bajos desempeños de los estudiantes.

Tabla 1.

Resultados del ANOVA para los niveles de comprensión por semestre académico de los alumnos

\begin{tabular}{|c|c|c|c|c|c|c|}
\hline $\begin{array}{l}\text { Nivel de } \\
\text { comprensión }\end{array}$ & $F$ & $g l 1$ & $g l 2$ & post hoc & Comparaciones por semestre & $\mathrm{p}$ \\
\hline Literal & 6.048 & 3 & 566 & Games-Howell & $\begin{array}{ll}1^{\text {ero }} M=0.86 & D E=0.24 \\
7^{\text {mo }} M=0.75 & D E=0.32\end{array}$ & $.047^{*}$ \\
\hline & & & & & $\begin{array}{ll}3^{\text {ero }} M=0.89 & D E=0.23 \\
7^{\text {mo }} M=0.75 & D E=0.32\end{array}$ & $.004^{* *}$ \\
\hline Inferencial & 3.452 & 3 & 566 & Bonferroni & $\begin{array}{ll}5^{\text {to }} M=0.73 & D E=0.28 \\
7^{\mathrm{mo}} M=0.62 & D E=0.32\end{array}$ & $.035^{*}$ \\
\hline Crítico & 3.774 & 3 & 566 & Bonferroni & $\begin{array}{l}1^{\text {ero }} M=3.0 \\
3^{\text {ero }} M=2.67\end{array}$ & $.043^{*}$ \\
\hline Apreciativo & 2.865 & 3 & 566 & Bonferroni & $\begin{array}{ll}5^{\text {to }} M=2.28 & D E=.602 \\
1^{\text {ero }} M=2.10 & D E=.547\end{array}$ & $.040^{*}$ \\
\hline
\end{tabular}

Nota. ${ }^{*} p<.05 ;{ }^{*} p<.01$ 
Otros datos importantes de la presente investigación se relacionan con la comparación de las ejecuciones en la prueba de comprensión lectora que mostraron los alumnos inscritos en distintos semestres de la Carrera de Psicología. Considerando el total del instrumento, no hay diferencias en la comprensión lectora de los alumnos de los cuatro semestres. Sin embargo, en el nivel de comprensión literal fueron mayores las habilidades mostradas por los estudiantes de los primeros semestres $\left(1^{\circ}\right.$ y $\left.3^{\circ}\right)$. Esto puede estar relacionado con la estructura del plan curricular universitario, que promueve en los primeros semestres una formación principalmente basada en aspectos teóricos, lo cual implica que el alumno tiene un mayor contacto con la lectura de libros y artículos para exponer en clase, para hacer monografías o ensayos, e incluso para presentar exámenes escritos; esta práctica puede fomentar la costumbre de extraer literalmente lo expuesto en un texto, y brinda una mayor oportunidad de desplegar estrategias lectoras para conseguir los objetivos académicos que le demandan sus profesores (lo cual puede relacionarse también con la mayor ejecución de los alumnos de primer semestre en el nivel crítico de comprensión).

En contraste, en los últimos semestres (de $5^{\circ}$ a $8^{\circ}$ ) la formación principalmente se enfoca en el módulo aplicado, lo que implica que la carga de trabajo recae en la realización de prácticas profesionales en escenarios naturales, tales como clínicas, escuelas, centros de rehabilitación e instituciones sociales; por ello las actividades académicas se relacionan con la elaboración de programas y reportes de actividades. Sin embargo, esta circunstancia no tendría por qué redundar en la disminución de sus habilidades de comprensión lectora.

Un elemento que puede ayudar a explicar los resultados de la presente investigación es el hecho de que no hay un fomento -curricular ni extracurricular- de habilidades relacionadas con la comprensión lectora; tampoco los contenidos curriculares están dirigidos a practicar y optimizar los distintos niveles de esta competencia. Al respecto, Cisneros et al. (2010) señalan que la preparación universitaria estrictamente disciplinar no impacta en los niveles de relación epistémica de los estudiantes con el texto científico-académico de corte expositivo-argumentativo. Por ello, según estos autores, después de los procesos de formación universitaria, los estudiantes no muestran herramientas de autorregulación, ni de metacognición en la lectura de textos académicos.

Los hallazgos de la presente investigación, aunados a lo reportado por Vera et al. (2010), respecto a la visión que tienen los alumnos en cuanto a sus propias competencias, $y$ al reconocimiento que hacen de su carencia de habilidades de abstracción, análisis, síntesis y crítica, aportan datos para ampliar el panorama de este complejo problema educativo.
Recapitulando, dicho panorama muestra, por un lado, que los programas de educación básica no parecen haber desarrollado en los alumnos niveles adecuados de competencia lectora (según lo demuestran las evaluaciones nacionales e internacionales expuestas); por otro, que los profesores universitarios se quejan de las escasas habilidades de comprensión lectora de sus alumnos, limitándose con ello el cumplimiento de sus objetivos de formación profesional; los propios alumnos reconocen conferirles poca importancia, aunque señalan que son escasos los esfuerzos de los programas académicos que sus universidades dirigen a fomentar el desarrollo de la abstracción y el análisis crítico de textos.

Una vista rápida de los objetivos formativos de cada grado escolar hace evidente que son los grados básicos los que deberían proveer a los alumnos de la competencia lectora en sus distintos tipos (o niveles de comprensión), y que están fallando en dicho cumplimiento. Los profesores universitarios podrían argumentar (y de hecho lo hacen) que no es su función desarrollar tales habilidades porque no son contemplados como objetivos específicos de los programas a nivel de licenciatura. Quienes diseñan los programas universitarios parten de la expectativa de que los alumnos ya deben contar con un pleno desarrollo de la competencia lectora para comprender los conceptos científicos y los componentes metodológicos que han de constituirse en elementos de su formación profesional. Y los alumnos pueden excusarse diciendo que no desarrollan habilidades lectoras complejas porque tendrían que ser autodidactas en ese proceso. Como salta a la vista, los problemas en el campo de la comprensión lectora de los alumnos universitarios no son atribuibles a un solo factor, sino que presentan muchas aristas. La pregunta obligada es: ¿Quién ha de romper con dicho círculo?

Lo que debe quedar claro a las instituciones educativas, así como a los alumnos y a los profesores (de todos los grados y niveles educativos), es que la solución debe aportarse desde distintos frentes. Es importante que las instituciones educativas tomen como base los datos disponibles, como los aportados por el presente estudio, para implementar cursos, programas, talleres, propedéuticos u otros recursos que promuevan en sus estudiantes competencias y prácticas efectivas para la comprensión lectora en todos sus niveles (literal, de organización de la información, inferencial, crítico y apreciativo). Para ello, es obvio que requieren preparación explícita y una guía puntual sobre cómo promover cada nivel, con diferentes tipos de texto, y abordando distintos temas y niveles de complejidad.

Edmonds et al. (2009) plantean que los estudiantes con problemas en la comprensión lectora pueden mejorar estas habilidades cuando se les enseñan explícitamente 
prácticas enfocadas en ellas. Los autores afirman que lo anterior parece obvio; sin embargo, a los estudiantes no se les enseñan adecuadamente las estrategias de comprensión lectora que requieren en los diferentes grados escolares. Todas estas inhabilidades de los estudiantes se acumulan en sus años de formación académica, y resulta paradójico que aún con esas deficiencias acrediten sus materias y logren incorporarse a la universidad, e incluso graduarse sin haber superado sus niveles de competencia. Todo esto porque se carece de programas efectivos de capacitación en materia de comprensión lectora.

En el caso específico de la formación de estudiantes universitarios, Serrano de Moreno (2008) menciona que ésta debe contemplar el entrenamiento en la lectura multidisciplinaria y crítica, con el fin de incrementar su capacidad de evaluar y construir juicios, para tomar decisiones y posturas con respecto a distintos temas. Este tipo de lectura puede propiciar el desarrollo de un pensamiento complejo en los alumnos, que les facilite incorporarse al mundo del conocimiento y les ayude a transformar su entorno.

Las instituciones de educación superior pueden echar mano de instrumentos como el aquí utilizado (que evalúa distintos niveles de comprensión lectora), que les pueden auxiliar en la realización de evaluaciones diagnósticas tempranas y oportunas. La aplicación de una evaluación de la comprensión lectora de todos los alumnos inscritos en una escuela universitaria hace posible planear, sistematizar y operar los medios idóneos para coadyuvar en una mejor formación académica. Algunas instituciones universitarias ya han incorporado en su plan de estudios módulos para desarrollar en los alumnos competencias genéricas, entre ellas, algunas relacionadas con la comprensión lectora. La Universidad de Los Lagos, en Chile incluyó un módulo denominado "competencias transversales para el aprendizaje", con dos créditos semanales y con carácter obligatorio (Quintana et al., 2007); mientras que en la Universidad Tecnológica de Pereira, Colombia, se elaboró una propuesta didáctica con el objetivo de potenciar en sus estudiantes la habilidad inferencial, involucrada en los procesos de comprensión de textos expositivos y argumentativos (Cisneros, Olave \& Rojas, 2012). Para ello, puede ser necesaria una capacitación sobre estrategias de comprensión lectora, dirigida a docentes universitarios, como lo señalan Martínez, Díaz y Rodríguez (2011).

\section{REFERENCIAS}

Amador, K. \& Alarcón, L. (2006). Propuesta metodológica para evaluar la comprensión lectora en estudiantes universitarios. Graffylia. Revista de la Facultad de Filosofia y Letras.
Benemérita Universidad de Puebla, 6, 126-135. Disponible en: http://www.filosofia.buap.mx/Graffylia/

Arroyo, R., Morales, G., Silva, H., Canales, C., \& Carpio, C. (2011). Evaluación de habilidades lectoras en la educación superior. En Irigoyen, J. J., Acuña K. \& Jiménez, M. (Coord.), Evaluación de Desempeños Académicos (pp. 97106). México: Universidad de Sonora.

Backhoff, E., Sánchez, A., Peón, \& Andrade, E. (2010). Comprensión lectora y habilidades matemáticas de estudiantes de educación básica en México: 2000-2005. Revista Electrónica de Investigación Educativa, 12(1). Disponible en: http://redie.uabc.mx/vol12no1/contenido-backhoffsanchez.html

Bazán, A., Castañeda, S., Macotela, S., \& López, M. (2004). Evaluación del desempeño en lectura y escritura. Aportes empíricos a la noción de componentes lingüísticos en el cuarto grado de primaria. Revista Mexicana de Investigación Educativa, 9(23), 841-861.

Calderón, A. \& Quijano, J. (2010). Características de comprensión lectora en estudiantes universitarios. Revista Estudios Socio-Jurídicos, 12(1), 337-364.

Cela, J. \& Ayala, F. (2001). Senderos de la evolución humana. Madrid: Alianza.

Cisneros, M., Olave, G., \& Rojas, I. (2010). La inferencia en la comprensión lectora: de la teoría a la práctica en la Educación Superior. Reseña de O. Silva (2010). Estudios Filológi$\cos , 45,129-130$. Disponible en: http://dx.doi.org/10.4067/ S0071-17132010000100012

Cisneros, M., Olave, G., \& Rojas, I. (2012). Cómo mejorar la capacidad inferencial en estudiantes universitarios. Educación y Educadores 15(1), 45-61.

Díaz, A. (2006). El enfoque de competencias en la educación. ¿Una alternativa o un disfraz de cambio? Perfiles Educativos, XXVIII (111), 7-36.

Echevarría, Ma. Á. \& Gastón, I. (2000). Dificultades de comprensión lectora en estudiantes universitarios. Implicaciones en el diseño de programas de intervención. Revista de Psicodidáctica, 10, 1-15.

Edmonds, S., Vaughn, S., Wexler, J., Reutebuch, C., Cable, A., Klingler, K., \& Wick, J. (2009). A synthesis of reading interventions and effects on reading comprehension outcomes for older struggling readers. Review of Educational Research, 79(1), 262-300.

Field, A. (2000). Discovering statistics using SPSS for Windows. Advanced techniques for the beginner. London: Sage Publications.

Goscinski, A., Campbell, M., Dew, R., Horan, P., Newlands, D., Rough, J., Silcock, J., \& Zhou, W. (2005). An IT bachelor degree using modern technologies to illustrate core concepts and principles and building generic skills. Education and Information Technologies, 10(4), 361-379.

Guerra, J. \& Guevara, Y. (2013). Validación de un instrumento para medir comprensión lectora en alumnos universitarios 
mexicanos. Enseñanza e Investigación en Psicología, 18 (2), 277-291.

Irigoyen, J., Acuña, K., \& Jiménez, M. (2010). Análisis de competencias académicas en la formación de estudiantes en ciencias. En M. T. Fuentes, J. J. Irigoyen \& G. Mares, Tendencias en psicología y educación. Revisiones temáticas (pp. 95-127). Volumen 1. México: Red Mexicana de Investigación en Psicología Educativa. Sistema Mexicano de Investigación en Psicología.

Irigoyen, J., Acuña, K., \& Jiménez, M. (2011). Interacciones didácticas en educación superior. Algunas consideraciones sobre la evaluación de desempeños. En Irigoyen, J. J., Acuña, K., \& Jiménez, M. (Coords.), Evaluación de desempeños académicos (pp. 73-96). México: Universidad de Sonora.

Llorens, A., Gil, L., Vidal, E., Martínez, T., Mañá, A., \& Gilabert, R. (2011). Prueba de competencia lectora para educación secundaria (CompLEC). Psicothema, 23(4), 808-817.

Martínez, E., Díaz, N., \& Rodríguez, D. (2011). El andamiaje asistido en procesos de comprensión lectora en universitarios. Educación y Educadores, 14(3), 531-556.

Méndez, I., Namihira, D., Moreno, L., \& Sosa, C. (2006). El protocolo de investigación. Lineamientos para su elaboración y análisis. México: Trillas.

Mujica, A., Guido, P., \& Mercado, S. (2011). Actitudes y comportamiento lector: una aplicación de la teoría de la conducta planeada en estudiantes de nivel medio superior. Liberabit, 17(1), 77-84.

Organización para la Cooperación y el Desarrollo Económico, OCDE. (2007). Pisa 2006: Aptitudes para las ciencias hacia el mundo del mañana. Nota informativa para México. Perfiles Educativos, XXIX (118), 99-105.

Pérez, M. J. (2005). Evaluación de la comprensión lectora: dificultades y limitaciones. Revista de Educación, número extraordinario, 121-138.

Quintana, M., Raccoursier, S., Sánchez, A., Sidler, H., \& Toirkens, J. (2007). Competencias transversales para el aprendizaje en estudiantes universitarios. Revista Iberoamericana de Educación, 44, 1-6.

Ramos, C. (2006). Elaboración de un instrumento para medir comprensión lectora en niños de octavo año básico. Onomázein, 14, 197-210.

Rizo, M. (2004). Programa de instrucción para desarrollar estrategias para la comprensión y el aprendizaje de textos escritos. Revista Latinoamericana de Estudios Educativos, 34(2), 113-137.

Sánchez, E. \& Acle, G. (2001). Relación entre comprensión lectora y niveles de pensamiento en estudiantes universitarios. Enseñanza e Investigación en Psicología, 6(2), 225-241.

Sánchez, A. \& Andrade, E. (2009). El aprendizaje en tercero de secundaria en México. Informe sobre los resultados del Excale 09, aplicación 2008. México: Instituto Nacional para la Evaluación de la Educación. Disponible en: http://www.inne.edu.mx/archivosbuscador/2008/01/INNE20080162-excale09completoa.pdf

Sánchez, S., Grajales, I., \& García, E. (2011). Un instrumento para el diagnóstico de habilidades de lectura de los estudiantes de la Licenciatura en Enfermería, UNSIS. Perfiles Educativos, XXXIII (132), 110-126.

Secretaría de Educación Pública, SEP. (2011). Documento base del bachillerato general. México: Secretaría de Educación Pública. Disponible en: http://www.dgb.sep.gob.mx/02$\mathrm{m} 1 / 03$-iacademica/01-programasdeestudio/documentobase/doc_base_032012_rev01.pdf

Secretaría de Educación Pública, SEP. (2013). Las competencias genéricas en el estudiante del bachillerato general. México: Secretaría de Educación Pública. Disponible en: http://www.dgb.sep.gob.mx/02-m1/03-iacademica/00otros/cg-e-bg.pdf

Serrano de Moreno, M. (2008). El desarrollo de la comprensión crítica en los estudiantes universitarios: hacia una propuesta didáctica. Educere, 42, 505-514.

Sumsion, J. \& Goodfellow, J. (2004). Identifying generic skills through curriculum mapping: A critical evaluation. Higher Education Research \& Development, 23(3), 329-346.

Ugarriza, N. (2006). La comprensión lectora inferencial de textos especializados y el rendimiento académico de los estudiantes universitarios del primer ciclo. Persona, 9, 31-75.

Vera, J., Estévez, E., \& Ayón, L. (2010). Percepción de estudiantes universitarios sobre importancia y realización de competencias genéricas. Revista de Educación y Desarrollo, 15, 47-54.

Walter, C. (2004). Transfer of reading comprehension skills to L2 is linked to mental representations of text and to L2 working memory. Applied Linguistics, 25(3), 315-339.

Zarzosa, L. (1997). La lectura y escritura en una población universitaria. Enseñanza e Investigación en Psicología, 2(1), 94-123. 